\title{
Effects of Multi-Directional Step-Up Training with Rhythmic Auditory Stimulation on Gait and Balance Ability in Stroke Patients
}

\author{
JIEUN CHOI ${ }^{1}$, JOONG-HWI KIM ${ }^{2}$ \\ ${ }^{1}$ Department of Physical Therapy, Graduate School, Daegu Catholic University \\ Hayang-Ro 13-13, Hayang-Eup, Gyeongsan-si, Gyeongbuk, REPUBLIC OF KOREA \\ ${ }^{2}$ Department of Physical Therapy, Daegu Catholic University \\ Hayang-Ro 13-13, Hayang-Eup, Gyeongsan-si, Gyeongbuk, REPUBLIC OF KOREA
}

\begin{abstract}
The purpose of this study was to investigate the effects of multi-directional step-up training with rhythmic auditory stimulation on gait and balance ability in stroke patients and also to help develop fall prevention and exercise programs for returning to daily life after stroke. The intervention method was randomly assigned to sixteen stroke patients, eight patients each in the experimental group and the control group. The experimental group carried out multi-directional step-up training with rhythmic auditory stimulation, and the control group performed only multi-directional step-up training. The training proceeded twelve times for 30 minutes for four weeks, and functional gait assessment, 10-meter walk test, and dynamic (by the Berg balance scale) and static (by Balancia software, Mintosys, Korea) balance ability assessments were conducted to examine the effect of the training on improving balance and gait ability. The study compared and analyzed the differences in the amount of change within the groups before and after training, and the differences in the amount of change between the two groups. Both groups showed more improved results after training than before in all assessments that measured gait and balance ability $(\mathrm{p}<0.05)$. However, the experimental group showed a greater difference in the amount of change in every gait and balance ability assessment compared to the control group. $(\mathrm{p}<0.05)$. Therefore, multi-directional step-up training can be an efficient intervention to improve the gait and balance ability of stroke patients and when accompanied by rhythmic auditory stimulation, the effectiveness could be maximized.
\end{abstract}

Key-words: - Stroke, rehabilitation, rhythmic auditory stimulation, balance training, gait

Received: February 14, 2021. Revised: July 1, 2021. Accepted: July 7, 2021. Published: July 15, 2021.

\section{Introduction}

The independent gait of stroke patients requires postural stability and balance ability. Decreases in the balance ability of stroke patients are indicated by abnormal muscle recruitment in the less-affected side due to muscle weakness in the affected side [1]. It is also caused by asymmetrical weight-bearing due to decreases in postural stability in static and dynamic standing. These balance impairments cause delaying motor skill recovery and gait disturbance and increase the incidence of falls [2].

Stroke patients can frequently experience falls due to uneven ground and obstacles indoors and outdoors during their daily activities. Stroke patients use strategies different than those of normal people to pass obstacles. They approach the obstacle closely and decrease gait speed to raise their feet as much as possible to ensure adequate space from obstacles [3].

Therefore, in the case of stroke patients, passing obstacles is a very complex lower extremity movement task, and requires the interaction between anticipatory postural control and voluntary movement.

Since the improvement in the balance ability of stroke patients is very important in performing gait and functional activities, various balance and gait training methods are used for stroke patients in clinical practice. Among them, there is a training method using Rhythmic Auditory Stimulation (RAS), which is known to effectively improve the gait disturbance of stroke patients by effectively stimulating the supraspinal region, and studies to identify its effectiveness have been conducted [4].

RAS training can exert an overall positive effect on gait performance as it improves the goal-oriented movement ability that adjusts footsteps according to the patient's timing and time according to a beat. It is assumed that the effect of RAS is caused by the control of auditory-motor coupling and 
corticostriatal activity. In particular, RAS recovers the auditory-motor coupling mechanism of neural networks, such as the cerebral cortex, cerebellum, supplementary motor area, and the premotor cortex in Parkinson's patients. Auditory-motor coupling is important not only for internal timing, which is associated with repetitive semi-automatic movements, sensory motors, and coordination mechanisms but upper extremity movements during gait performance [5].

In addition, training using stepping or stepping over obstacles can maximize the effect of motor learning by exposing stroke patients to unfamiliar and difficult environments and directly stimulating the central nervous system [6]. The square step-up training in which patients step-up on the box in several directions had a more significant effect in improving lower extremity strength and balance ability than the general walking exercise group [7]. When it comes to improving gait ability, stair stepup training increased the activity of the rectus femoris and improved weight-bearing to the affected side, showing a positive effect on stability during the stance phase.

Stroke also decreases cognitive ability, and poststroke patients suffer from impaired concentration, attention, and executive function when they have to perform both motor and cognitive tasks at the same time even when they return to their daily lives [8-9]. Therefore, it is important to train using cognitive task feedback along with motor tasks in the rehabilitation intervention for the return to daily life after stroke [10].

Among the cognitive tasks, auditory stimulation shows powerful effects, so even though information on the visual target was provided in advance, the subjects of the experiment focused on unexpected sounds reflexively. Thus, the surrounding auditory stimulus was a more powerful factor than attracting visual attention [11-12]. Therefore, if these auditory stimuli are used for gait and balance training for stroke patients, it will help the patient to perform exercise and cognitive tasks simultaneously by enhancing concentration and attention in various circumstances besides in a clinic.

Previous studies described the necessity of stepup training and stair gait training to improve functional activity as well as gait and balance ability in stroke patients and proved their effectiveness. However, insufficient studies that have applied combining stepping exercise and RAS simultaneously. This study explored a training method to maximize the efficiency and concentration for improving patients' gait and balance ability without space, time, and economic constraints using simple tools rather than expensive equipment.

\section{Methods \\ 2.1 Subjects}

In this study, 16 post-stroke patients in a rehabilitation hospital voluntarily participated in the study after receiving sufficient explanation on the method and purpose of the study, which was approved by the Daegu Catholic University Institutional Review Board (CUIRB-2020-0072). The number of subjects was determined using the G-power program by setting the same number of experimental groups and control groups with a high effect size at the significance level of 0.5 for the independent sample t-test.

The participants were randomly divided into two groups. The experimental group was composed of $65.5 \%$ of males and $37.5 \%$ of females. Their average age was $49.88 \pm 8.77$ years old, their average height was $166.50 \pm 8.65 \mathrm{~cm}$, and Their average Mini-Mental State Examination-Korean version (MMSE-K) score was $26.38 \pm 2.77$ points. The control group was composed $65.5 \%$ of males and $37.5 \%$ of females. Their average age was 58.50 \pm 15.58 years old, their average height was 162.13 $\pm 11.65 \mathrm{~cm}$, and their average MMSE-K score was $26.88 \pm 2.42$ points. Since there was no significant difference in the general characteristics of the study subjects $(p>0.05)$, the homogeneity of the general characteristics between the groups was confirmed.

\subsection{Experimental Procedure}

As a multi-directional step-up training tool, three step-up boxes with a height of $10 \mathrm{~cm}, 20 \mathrm{~cm}$, and 10 $\mathrm{cm}$ were used, with a 10 height difference between the adjacent boxes. The experimental group was provided RAS during multi-directional step-up training. The Metronome beat from Soundbrenner (C2018 Soundbrenner), which is a metronome application on a smartphone, served as the RAS. Before the experiment, the participants performed 1 minute step-up training for five times to determined and record the average beats per minute (BPM) they felt comfortable with. The control group executed multi-directional step-up training without RAS. In both groups, 10 minutes of warm-up training including posture alignment and weight bearing 
training was performed, and then step-up training was done for the remaining 20 minutes. The training was carried out three times per week for four weeks. The detailed multi-directional step-up training procedure was as follows:

The position of the step box was arranged differently depending upon left or right hemiplegia. At first, the left hemiplegic participants turned clockwise from the starting point 1 (forward stepup, first, using the less-affected side) in the order of 2 (side step-up), 3 (backward step-down, first, using the affected side), and 4 (side step-down), and then returned to the starting point 1 . After that, they turned counterclockwise in the order of 4 (side stepup), 3(forward step-up, first using the less-affected side), and 2 (side step-down) and then returned to the starting point 1 (backward step-down, first, using the affected side) (Figure 1).
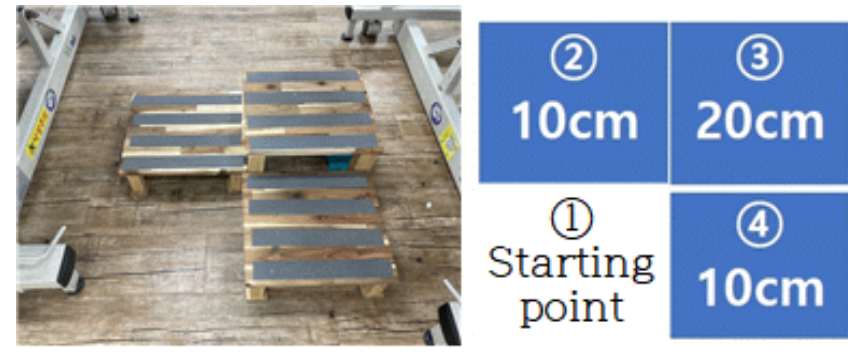

Fig.1: Step box layout for left hemiplegia

During the step-up training, the previously recorded BPM was provided as RAS for 20 minutes through a Bluetooth speaker. As the patient's skill level increased, the BPM was increased by $5-10 \%$ each week. During training, when the participants complained of fatigue or dizziness, they took a break to minimize the risk of falls.

\subsection{Evaluation}

To determine the differences in the amount of change in the evaluation results between the groups and within the groups before and after the experiment, all participants took the same balance and gait tests before and after the intervention. The gait ability tests included the functional gait evaluation (FGA) and the 10-meter gait test (10mWT). The berg balance scale (BBS) was used to measuring dynamic standing balance ability. For measuring static standing balance ability, Balancia which is a standing balance assessment software, was used to measure the average sway velocity average (Velocity AVG), the total sway path length(Path length), and the 95\% sway area (Area $95 \%)$.

\subsection{Statistical Analyses}

Analysis of the data collected in this study was done by the SPSS ver. 26 statistical program and normality was tested by the Kolmogorov-Smirnov test. To analyze the differences in the general characteristics of the two groups, an independent ttest was done, and differences in the amount of change within the groups before and after the experiment were analyzed by a paired t-test. An independent sample t-test was performed to compare and analyze the data between the groups. All statistical significance levels were set to $\mathrm{p}<$ 0.05 .

\section{Results}

To confirm the homogeneity between the experimental group and the control group in each test, we examined whether there were statistically significant differences in any variables for FGA, 10mWT, BBS, Velocity AVG, Path length, and Area $95 \%$ between the groups. There was no statistically significant difference in any tests $(\mathrm{p}>$ 0.05) (Table 1).

Table 1. Pre-homogeneity test for dependent variables

\begin{tabular}{|c|c|c|c|c|c|c|}
\hline & $\begin{array}{l}\text { Experi- } \\
\text { mental } \\
\text { group }\end{array}$ & & $\begin{array}{l}\text { Control } \\
\text { group }\end{array}$ & & $\mathbf{t}$ & $\mathbf{p}$ \\
\hline $\begin{array}{l}\text { FGA } \\
\text { (score) }\end{array}$ & $\begin{array}{l}12.37 \\
7.48^{\mathrm{a}}\end{array}$ & \pm & $\begin{array}{l}18.50 \\
8.25\end{array}$ & \pm & $\begin{array}{l}- \\
1.5 \\
6\end{array}$ & $\begin{array}{l}0.1 \\
4\end{array}$ \\
\hline $\begin{array}{l}10 \mathrm{mWT} \\
(\mathrm{sec})\end{array}$ & $\begin{array}{l}18.35 \\
11.06 \\
\end{array}$ & \pm & $\begin{array}{l}17.81 \\
12.41 \\
\end{array}$ & \pm & $\begin{array}{l}0.0 \\
9 \\
\end{array}$ & $\begin{array}{l}0.9 \\
3 \\
\end{array}$ \\
\hline $\begin{array}{l}\text { BBS } \\
\text { (score) }\end{array}$ & $\begin{array}{l}44.88 \\
6.40\end{array}$ & \pm & $\begin{array}{l}47.13 \\
8.15\end{array}$ & \pm & $\begin{array}{l}- \\
0.6 \\
1\end{array}$ & $\begin{array}{l}0.5 \\
5\end{array}$ \\
\hline $\begin{array}{l}\text { Velocit } \\
\text { y AVG } \\
(\mathrm{cm} / \mathrm{s})\end{array}$ & $\begin{array}{l}2.40 \\
0.46\end{array}$ & \pm & $\begin{array}{l}3.19 \\
0.82\end{array}$ & \pm & $\begin{array}{l}- \\
2.3 \\
7\end{array}$ & $\begin{array}{l}0.4 \\
4\end{array}$ \\
\hline $\begin{array}{l}\text { Path } \\
\text { length } \\
(\mathrm{cm})\end{array}$ & $\begin{array}{l}23.99 \\
4.62\end{array}$ & \pm & $\begin{array}{l}27.25 \\
3.88\end{array}$ & \pm & $\begin{array}{l}- \\
1.5 \\
2\end{array}$ & $\begin{array}{l}13 . \\
60\end{array}$ \\
\hline $\begin{array}{l}\text { Area } \\
95 \% \\
\left(\mathrm{~cm}^{2}\right)\end{array}$ & $\begin{array}{l}1.39 \\
1.20\end{array}$ & \pm & $\begin{array}{l}2.13 \\
1.40\end{array}$ & \pm & $\begin{array}{l}- \\
1.1 \\
4\end{array}$ & $\begin{array}{l}13 . \\
70\end{array}$ \\
\hline
\end{tabular}

${ }^{\mathrm{a}}$ mean \pm SD

In this study, FGA and 10mWT were used to evaluate the change in gait ability of stroke patients before and after the experiment. In the FGA, the score in the experimental group significantly increased from $12.37 \pm 7.48$ to $25.62 \pm 4.24$ after 
the training $(\mathrm{p}<0.05)$, and the score in the control group also significantly increased from $18.50 \pm 8.25$ to $23.32 \pm 4.59$ after the training $(\mathrm{p}<0.05)$. The difference in the amount of change between the two groups was $8.5 \pm 1.03$, showing a higher score in the experimental group with step-up training with RAS, but no statistically significant difference $(p>0.05)$.

To examine the difference in balance ability of the participants, BBS was measured before and after the intervention within the groups. The experimental group showed a significant score improvement from $44.87 \pm 6.40$ to $51.00 \pm 3.70(\mathrm{p}<0.05)$. The control group also showed a significant score improvement from $47.12 \pm 12.41$ to $48.75 \pm 8.45(\mathrm{p}<0.05)$. The difference in the amount of change between the two groups was $4.5 \pm 2.78$. The score improvement was significantly greater in the experimental group $(\mathrm{p}<$ $0.05)$.

Amount of change in Velocity AVG, Path length, and Area $95 \%$ by Balancia showed statistically significant reductions in both groups after training ( $p<0.05$ ) (Table 2). However, a significantly greater degree of decrease was seen in the experimental group compared to the control group $(\mathrm{p}<0.05) \quad($ Table 3)

Table 2. Comparison of the static standing balance ability measurements within the groups pre and postintervention

\begin{tabular}{llllll}
\hline & Groups & Pre & \multicolumn{1}{c}{ Post } & t & p \\
\hline \multirow{2}{*}{ Velocity AVG $(\mathrm{cm} / \mathrm{s})$} & Experimental group & $2.40 \pm 0.46^{\mathrm{a}}$ & $1.37 \pm 0.28$ & 6.36 & 0.05 \\
\cline { 2 - 6 } & Control group & $3.18 \pm 0.82$ & $2.77 \pm 0.52$ & 1.91 & 0.09 \\
\hline \multirow{2}{*}{ Path length $(\mathrm{cm})$} & Experimental group & $23.99 \pm 4.62$ & $14.95 \pm 3.28$ & 11.13 & $0.00^{*}$ \\
\cline { 2 - 6 } & Control group & $27.24 \pm 5.14$ & $24.22 \pm 5.14$ & 2.42 & 0.05 \\
\hline \multirow{2}{*}{ Area 95\% (cmin $)$} & Experimental Group & $1.38 \pm 1.20$ & $0.51 \pm 0.31$ & 2.75 & 0.05 \\
\cline { 2 - 6 } & Control group & $2.13 \pm 1.39$ & $1.97 \pm 1.34$ & 2.53 & 0.05 \\
\hline
\end{tabular}

${ }^{\mathrm{a}}$ mean $\pm \mathrm{SD},{ }^{*} \mathrm{p}<0.05$

Table 3. Comparison of $\Delta$ (mean difference) in the static standing balance ability measurements between the groups

\begin{tabular}{lllll}
\hline & Experimental group & Control group & t & p \\
\hline$\Delta$ Velocity AVG. $(\mathrm{cm} / \mathrm{s})$ & $1.02 \pm 0.45^{\text {a }}$ & $0.4 \pm 0.60$ & 2.29 & $0.00^{*}$ \\
\hline$\Delta$ Path length $(\mathrm{cm})$ & $9.03 \pm 2.30$ & $3.02 \pm 3.53$ & 4.03 & $0.00^{*}$ \\
\hline$\Delta$ Area $95 \%\left(\mathrm{~cm}^{2}\right)$ & $0.87 \pm 0.90$ & $0.16 \pm 0.18$ & 2.17 & 0.05 \\
\hline${ }^{\mathrm{a}}$ mean $\pm \mathrm{SD},{ }^{*} \mathrm{p}<0.05$ & & & &
\end{tabular}

\section{Discussion}

The purpose of this study was to explore the effectiveness of multi-directional step-up training with RAS for four weeks to improve the gait and balance ability of stroke patients. Through this study, we identified an effective treatment method that can help these patients improve their function of in clinical practice.

In stroke patients, a postural imbalance is aggravated by impaired balance control and increased postural agitation due to hemiplegic symptoms [12]. This postural imbalance also affects gait impairment by causing decreased gait speed and instability [13]. Due to the dysfunction in balance and gait ability, despite returning to daily life, patients could experience a lower quality of life and an higher risk of falling [14-15]. Therefore, it is important that stroke patients have frequent opportunities to practice task-oriented dynamic training, and that such training is effective for returning to daily life quickly, rebuilding independence, and improving the quality of life [16].

In this study, as a result of performing the multi-directional step-up exercise as part of taskoriented balance training, the BBS scores of all participants were increased after the intervention. In addition, balance ability was improved in both groups after training compared to before training in the Velocity AVG, the Path length, and the Area 95\% measurements made with the Balancia.

Since there is a high correlation between the balance and gait ability of stroke patients, it is essential to train balance ability such as developing postural stability and weight bearing control which could eventually improve gait ability as well [17]. In this study, FGA and the 10mWT were also carried out to determine any change in gait ability after balance training. In the FGA, both groups showed an increase in scores after the experiment, 
and in the $10 \mathrm{mWT}$, the walking times were shortened after the experiment in both groups.

These results could roughly be attributed to two factors. First, through multi-directional step-up training, both the affected and the less-affected low extremity joints alternately make dynamic movements, which could contribute to improving weight shifting in various directions that were limited after stroke. Thereby, it could be assumed that the level of ability on both sides was improved and the trunk and lower limbs were automatically controlled.

The second factor can be found in a previous study of step box and stair training in stroke patients. In that study, there were significant changes in muscle strengthening in the rectus femoris, tibialis anterior, and calf muscles in both groups that performed the step-up exercise[18]. This could also explain the results in this study showing significant improvements in gait and balance ability in both groups

Apart from step-up training, there have been many studies showing the effect of sensory feedback on motor learning training by providing visual or auditory stimulation to stroke patients [19-23]. To verify the effect of RAS for improving gait and balance ability, this study analyzed the difference between the experimental group with RAS and the control group without RAS

When comparing the two groups before and after the experiment, there was no significant difference between the two groups in the FGA, which measured the change in walking ability. However, in the 10mWT, the walking time was shorter in the experimental group than in the control group. A previous study in stroke patients also showed an improvement in walking speed in the experimental group that performed gait training with applied RAS. The study described that the auditory stimulation effectively stimulated the central pattern generator, eventually leading to improvements [24].

In the BBS, which was used to measure the change in dynamic standing balance before and after the experiment between the two groups, the experimental group showed higher scores than the control group, and there was a difference in the amount of change between the two groups. Furthermore, in static standing balance ability evaluated by the Balencia, the experimental group had a greater difference in the amount of change than the control group. Consequently, there was a significant improvement in gait and balance ability in the experimental group provided RAS than in the control group, which exercised with only step-up training without any auditory stimulation.

Previous studies explained that improved motor performance could be possible because repetitive auditory stimulation stimulates the rhythmic movements of patients with a damaged central nervous system [25]. Moreover, rhythmic auditory stimulation compensates for the nerve delay of the skin sensory receptors of the foot by simultaneously stimulating the central pattern generator and feedback at the level of the upper spinal cord through the reticulospinal tract, predicting the contact of the foot and adjusting the muscle activity before the foot even touches the ground. Through this process, a quick adaptation to the discrepancy between the peripheral sensory input (foot contact) and the upper spinal sensory input is made (auditory rhythm) [26].

In other words, it can be estimated that RAS provided repeatedly to the experimental group activated the cerebellum, corrected the repeated errors, and optimized the movement, thereby effectively improving the gait and balance skill compared to the control group.

\section{Conclusion}

Although this experiment showed that more efficiently improved balance and gait ability when multi-directional step-up training was accompanied by RAS compared to performing only multi-directional step-up training in stroke patients, the results are somewhat challenging to generalize to all stroke patients because of the insufficient number of participants. Since the experiment was conducted for four weeks, it is difficult to determine the long-term effect.

Therefore, more follow-up studies are needed to generalize and scientifically prove the effect of multi-directional step-up exercise with RAS. Nevertheless, this study showed the possibility that incorporating RAS into step-up training could be part of a rehabilitation program for stroke patients that could effectively improve balance skills by maximizing efficiency and concentration.

\section{References:}

[1] K. L. Harburn, K. M. Hill, "Clinical applicability and test-retest reliability of an external perturbation test of balance in stroke subjects," Arch Phys Med Rehabil, Vol. 76, 1995, pp. 317-23. 
[2] L. Nyberg, Y. Gustafson, "Patient Falls in Stroke Rehabilitation: A Challenge to Rehabilitation Strategies," Stroke, Vol. 26, No. 5, , 1995, pp. 838842.

[3] H. C. Chen, J. A. Ashton-Miller, "Stepping over obstacles: gait patterns of healthy young and old adults," Journal of Gerontology, Vol. 46, No. 6, 1991, pp. 196-203.

[4] M. H. Thaut, K. M Stephan, "Distinct corticocerebellar activations in rhythmic auditory motor synchronization," Cortex, Vol. 45, No. 1, 2009, pp. 44-53.

[5] A. Naro, L. Pignolo, "A Case-Controlled Pilot Study on Rhythmic Auditory Stimulation-Assisted Gait Training and Conventional Physiotherapy in Patients with Parkinson's Disease Submitted to Deep Brain Stimulation," Front. Neurol, Vol. 11, No. 794, 2020.

[6] E. Kloter, M. Wirz, "Locomotion in stroke subjects: interactions between unaffected and affected sides," Brain, Vol. 134, 2011, pp.721-731.

[7] R. Shigematsu, T. Okura, "Square-stepping exercise and fall risk factors in older adults: a single-blind, randomized controlled trial," $J$ Gerontol A Biol Sci Med Sci, Vol. 63, No. 1, 2008, pp. 76-82.

[8] A. Bowen, R. Wenman, "Dual-task effects of talking while walking on velocity and balance following a stroke," Age Ageing, Vol. 30, No. 4, 2001, pp. 319-23.

[9] L. Abu-Qatouseh, E. Mallah, M. Bustami, E. Alkhateeb, "Increased Resistance of Fluoroquinolones among H. pylori Isolates from Patients with Gastroduodenal Diseases in Jordan", WSEAS Transactions on Biology and Biomedicine, 2020, pp. 76-81, Volume 17.

[10] J. A. Michel, C. A. Mateer, "Attention rehabilitation following stroke and traumatic brain injury", A review Eura Medicophys, Vol. 42, No. 1, 2006, pp. 59-67.

[11] V. Mazzaa, M. Turatto, "How automatic are audiovisual links in exogenous spatial attention?" Neuropsychologia, Vol. 45, 2007, pp. 514-522.

[12] I. S. Mavromatakis, S. G. Liliopoulos, G. S. Stavrakakis, "Optimized Intermittent Pharmaceutical Treatment of Cancer using NonLinear Optimal Control Techniques", WSEAS Transactions on Biology and Biomedicine, Volume 17, 2020, pp. 67-75.

[13] Y. Laufer, R. Dickstein, "The effect of treadmill training on the ambulation of stroke survivors in the early stages of rehabilitation: a randomized study," J Rehabil Res Dev, Vol. 38, No. 1, 2001, pp. 69-78.

[14] K. Dodd, M. E. Morris, "Lateral pelvic displacement during gait: abnormalities after stroke and changes during the first month of rehabilitation," Arch Phys Med Rehabil, Vol. 84, No. 8, 2003 pp. 1200-1205.

[15] P.Y. Lin, Y. R. Yang, "The relation between ankle impairments and gait velocity and symmetry in people with stroke," Arch Phys Med Rehabil, Vol. 87, No.4, 2006, pp. 562-568.

[16] N. M. Salbach, N. E. Myo, "Balance self-efficacy and its relevance to physical function and perceived health status after stroke," Arch Phys Med Rehabil, Vol. 87, No. 3, 2006, pp. 364-370.

[17] M. A. Keenan, J. Perry, "Factors affecting balance and ambulation following stroke," Clin Orthop Relat Res, Vol. 182, 1984, pp. 165-171.

[18] H. S. Kim, “The Effect of Type of Treads for Step Training on Trunk and Lower Extremity Muscle Activity and Balance of Chronic Stroke Patients," Daegu University Graduate School doctoral dissertation, 2015.

[19] C. B. Cha, R. C. O'connor, “Testing moodactivated psychological markers for suicidal ideation," Journal of Abnormal Psychology, Vol. 127, No. 3, 2018, pp. 448-457.

[20] M. E. Khallaf, A. M. Gabr, "Effect of Task Specific Exercises, Gait Training, and Visual Biofeedback on Equinovarus Gait among Individuals with Stroke: Randomized Controlled Study," Neurol Res Int. 2014, pp. 1-9.

[21] S. Mainka, J. Wissel, "The Use of Rhythmic Auditory Stimulation to Optimize Treadmill Training for Stroke Patients: A Randomized Controlled," Front. Neurol, Vol. 9, No. 755, 2018.

[22] Y. R. Yang, Y. H. Chen, "Effects of interactive visual feedback training on post-stroke pusher syndrome: a pilot randomized controlled study," Clin Rehabil, Vol. 29, No. 10, 2015, pp. 987-993.

[23] S. Fendrihan, M. C. Popescu, "Cluster Analysis of Candida Infections Evolution in Urban and Rural Areas in Romania", WSEAS Transactions on Biology and Biomedicine, Volume 17, 2020, pp. 58-66.

[24] T. A. Pelton, L. Johannsen, "Hemiparetic stepping to the beat: Asymmetric response to metronome phase shift during tread mill gait," Neurorehabil Neural Repair, Vol. 24, No. 5, 2010, pp. 428-434.

[25] M. H. Thaut, G. P. Kenyon, "Rapid motor adaptations to subliminal frequency shifts during syncopated rhythmic sensorimotor synchronization," Hum Mov Sci, Vol. 22, No. 3, 2003, pp. 321-338.

[26] A. Forner-Cordero, J. P. Pinho, "Effects of supraspinal feedback on human gait: rhythmic auditory distortion," J Neuroeng Rehabil, Vol. 16, No. 159, 2019.

Creative Commons Attribution License (Attribution 4.0 International, CC BY 4.0)

This article is published under the terms of the Creative Commons Attribution License 4.0 https://creativecommons.org/licenses/by/4.0/deed.en_US 